\title{
Cardiopulmonary responses to exercise in an individual with Myalgic Encephalomyelitis/Chronic Fatigue Syndrome during long-term treatment with intravenous saline: A case study
}

\author{
Todd E. Davenport*,a,b, Michael K. Ward ${ }^{\mathrm{a}}$, Staci R. Stevens ${ }^{\mathrm{b}}$, Jared Stevens ${ }^{\mathrm{b}}$, \\ Christopher R. Snell ${ }^{\mathrm{b}}$ and J. Mark VanNess ${ }^{\mathrm{b}, \mathrm{c}}$ \\ ${ }^{a}$ Department of Physical Therapy, School of Health Sciences, University of the Pacific, Stockton, CA, USA \\ ${ }^{\mathrm{b}}$ Workwell Foundation, Ripon, CA, USA \\ ${ }^{\mathrm{c}}$ Department of Health, Exercise, and Sport Science, College of the Pacific, University of the Pacific, \\ Stockton, CA, USA
}

Received 13 September 2019

Accepted 29 March 2020

\begin{abstract}
BACKGROUND: Myalgic Encephalomyelitis/Chronic Fatigue Syndrome (ME/CFS) causes significant impairment in daily activities, including the ability to pursue daily activities. Chronotropic intolerance is becoming better characterized in ME/CFS and may be the target of supportive treatment.

OBJECTIVE: To document the effect of repeated intravenous (IV) saline administration on cardiovascular functioning and symptoms in a 38-year old female with ME/CFS.

METHODS: The patient received $1 \mathrm{~L}$ of $0.9 \%$ IV saline through a central line for a total of 675 days. Single CPETs were completed periodically to assess the effect of treatment on cardiopulmonary function at peak exertion and ventilatory anaerobic threshold (VAT). An open-ended symptom questionnaire was used to assess subjective responses to CPET and self-reported recovery time.

RESULTS: Improvements were noted in volume of oxygen consumed $\left(\mathrm{VO}_{2}\right)$, heart rate (HR), and systolic blood pressure (SBP) at peak and VAT. Self-reported recovery time from CPET reduced from 5 days to 1-2 days by the end of treatment. The patient reported improved quality of life related, improved capacity for activities of daily living, and reduced symptoms. CONCLUSIONS: IV saline may promote beneficial effects for cardiopulmonary function and symptoms in people with ME/CFS, which should be the focus of formal study.
\end{abstract}

Keywords: Treatment, exercise testing, Myalgic Encephalomyelitis, Chronic Fatigue Syndrome

\footnotetext{
*Address for correspondence: Dr. Todd E. Davenport. E-mail: tdavenport@pacific.edu.
} 


\section{Introduction}

Myalgic Encephalomyelitis/Chronic Fatigue Syndrome (ME/CFS) is a seriously disabling health condition characterized by myalgia, cognitive impairment, post-exertional malaise (PEM), potential mood disruption, and persistent mental and physical fatigue. The hallmark clinical feature of ME/CFS is PEM [1, 2]. PEM involves a constellation of severely disabling signs and symptoms in response to exertion. PEM imposes a significant, life-altering burden on patients, impairing their ability to tolerate activities of daily living and working that are necessary to conduct life at pre-illness levels of functioning. Among patients with fatiguing health conditions, ME/CFS causes the most severe self-reported functional deficits [3, 4]. Perhaps not surprisingly, ME/CFS commonly leads to the inability to maintain remunerative employment $[5,6]$, which is associated with a loss of personal income [3] and reliance on socialized economic supports. Further, the social stigma of an 'invisible disability' may compound functional inability and financial impacts, because ME/CFS may not be adequately recognized and supported by employers [7]. Thus, interventions appropriately aimed at reducing symptoms and improving function can ameliorate a spiraling cycle of symptomatic, functional, and financial decline.

The pervasive nature of PEM in ME/CFS has led some working groups to revise diagnostic criteria for ME/ CFS to highlight the multisystem deficits associated with exertional intolerance [1]. An emerging hypothesis holds that symptoms and functional disability from ME/CFS involves neuroinflammation, resulting in damage of both peripheral and central nervous structures [8-10]. This damage to peripheral and central nervous system structures, particularly the involvement of central autonomic cardiac control centers $[9,10]$, may explain the observed abnormal cardiac regulation in ME/CFS, including diminished blood volume and orthostasis. In support of this hypothesis, a recent systematic review identified abnormal chronotropic responses to exercises in people with ME/CFS compared to matched control subjects [11]. Therefore, support for cardiac functioning may improve symptoms and functional decrements associated with ME/CFS, and improve the ability for daily activities including work.

Intravenous (IV) saline may ameliorate abnormal cardiac responses by temporarily increasing vascular volume, which increases cardiac output through three primary pathways: Increases in vascular volume stimulate juxtaglomerular cells, increasing renin production, which elevates circulating angiotensin II. Angiotension II increases perfusion pressure by inducing systemic vasoconstriction. Central angiotensinergic activity may also stimulate the paraventricular hypothalamus, increasing sympathetic drive. Finally, increased end-diastolic volume as a result of increased vascular volume causes increased cardiac contractility via the FrankStarling mechanism. The net effect is an increase in cardiac output as a result of increased contractility and sympathetic drive. This intervention has already shown to improve resting hemodynamics in patients with postural orthostatic tachycardia syndrome (POTS), which is a disease characterized by decreased stroke volume and abnormally elevated heart rate in response to positional changes. This observation seems notable because POTS is also a common comorbidity with ME/CFS [12].

The purpose of this case study is to investigate the effect of repeated IV saline administration on symptoms and functioning in a patient with ME/CFS. For this case study, functioning was measured on the basis of a single graded exercise test, which was completed periodically during treatment.

\section{Case description}

\subsection{Case history}

The patient was a 38 year old female who was diagnosed with ME/CFS by an internal medicine physician according to the International Consensus Criteria [1]. She first experienced symptoms as a result of a mononucleosis infection at age 21, and did not recover functionally. Prior to the onset of ME/CFS symptoms, the patient's occupation was a full-time college student and intercollegiate basketball player, who enjoyed many types of physical activity and vigorous exercise. As her condition declined, her primary symptoms included "brain fog," profound tiredness following prolonged upright positioning, and hallmark signs of PEM characteristic of ME/CFS. Of note, the patient had chronically low blood pressure. However, the patient did not experience noticeable tachycardia, chest pains, dizziness, and lightheadedness or syncope during positional changes, which are characteristic of POTS.

Following the development of more severe symptoms, the patient had to forego nearly all forms of 
sustained aerobic activity. The patient was capable of interval-type activity with an emphasis on strength and functional movements. She managed her symptoms by scheduling rest prior to and following any physically or mentally demanding events. Adept energy conservation and symptom management allowed the patient to participate in community volunteering and to assist in childcare at home, but this functional level was not sufficient to allow a return to remunerative work in community settings. At the time of treatment, the patient had adopted a living environment that allowed for energy conservation strategies sufficient for intermittent activity. She had educated family members and peers about her condition and has been met with support and sympathy. Despite her efforts, she still experienced periods of symptom exacerbation that significantly limited her functioning.

\subsection{Intervention and follow-up measurements}

A central venous line was inserted into the superior vena cava by an interventional radiologist. The patient then received $1 \mathrm{~L}$ of $0.9 \%$ saline solution daily for 678 days by way of the central venous line. Responses to treatment were recorded through maximal cardiopulmonary exercise testing (CPET), which was performed at days $15,55,92,125,180,248,317$, 420 , and 675 , in order to assess cardiac, pulmonary, and metabolic functioning during the course of treatment. The test intervals were pragmatically in an attempt to balance the need for objective information about physiological functioning with the availability of the patient, availability of testers, and the risk for exacerbating long-term PEM. An additional exercise test was administered on day 708 , thirty days after the patient's final infusion, in order to assess the physiological effects of withdrawing treatment. Single test methodology was selected to reduce the symptomatic and functional impacts associated with serial CPET, because the plan for follow-up testing involved frequent maximal CPET [13]. As such, these physiological measurements should be interpreted as changes in baseline CPET measures over time, rather than the effects of PEM or physiological ability to recover from a CPET challenge.

The patient periodically completed an exercise challenge consisting of a single peak CPET on an electronically braked cycle ergometer [13-17]. Before the exercise test, the entire procedure for exercise testing was explained in detail. The patient was fitted with 10-lead electrocardiography elec-
Table 1

Open-ended questions used to assess recovery immediately after cardiopulmonary exercise testing (CPET), 7 days following CPET, and before the subsequent CPET

- How did you feel following the exercise test?

- Describe how you felt the day after the exercise test.

- How long did it take you to recover from the exercise test? Circle the appropriate time (in days).

- Describe symptoms, if any, experienced after the exercise test.

trodes for monitoring of heart rhythm, a mask, and headgear for collection of expired air, and a pulse oximeter for monitoring arterial oxygen saturation. The patient was allowed to pedal for a short period (less than one minute). Workload was increased 15 watts per minute until voluntary exhaustion. ECG was monitored continuously for signs of cardiac arrhythmia or ischemia, and pulse oximetry was monitored to ensure safe levels of arterial oxygenation. The patient was encouraged to pedal as long as possible, and testing was terminated when criteria for peak effort were met [18]. Test termination criteria consisted of meeting two cessation criteria, including of an observed plateau in oxygen consumption with increases in workload, RPE $\geq 18(6-20$ scale), and respiratory exchange ratio (RER) $\geq 1.1$ [18]. The patient then remained seated on the ergometer and recovery was monitored for 2-5 minutes.

Throughout testing, measurements were taken at peak to assess the volume of oxygen consumed $\left(\mathrm{VO}_{2}\right)$, minute ventilation $\left(\mathrm{V}_{\mathrm{E}}\right)$, peak heart rate (HR), peak systolic blood pressure (SBP), respiratory exchange ratio (RER), and workload. Additionally, $\mathrm{VO}_{2}$, workload, and HR were assessed at ventilatory anaerobic threshold (VAT), which was defined as the respiratory compensation point. The patient completed an open-ended recovery questionnaire to record subjective responses to CPET and recovery time (Table 1), immediately after the test, 7 days after the test, and then again prior to the subsequent test.

\section{Case outcomes}

Post hoc assessment of CPET results indicates that daily saline infusions were associated with improvements in cardiopulmonary responses on CPET (Table 2). Peak $\mathrm{VO}_{2}$ showed a general linear improvement from $23.7 \mathrm{ml} / \mathrm{kg} / \mathrm{min}$ ( $72 \%$ of age- 
Table 2

Cardiopulmonary exercise test measurements obtained at peak exertion and ventilatory anaerobic threshold throughout treatment with intravenous saline

\begin{tabular}{|c|c|c|c|c|c|c|c|c|c|c|c|}
\hline \multirow{2}{*}{$\begin{array}{l}\text { Days Post- } \\
\text { Infusion }\end{array}$} & \multicolumn{2}{|c|}{$\mathrm{VO}_{2}$ Peak $(\mathrm{ml} / \mathrm{kg} / \mathrm{min})$} & \multirow[t]{2}{*}{$\mathrm{V}_{\mathrm{E}}(\mathrm{L} / \mathrm{min})$} & \multicolumn{2}{|c|}{ HR Peak (beats/min) } & \multirow{2}{*}{$\begin{array}{c}\text { SBP Peak } \\
(\mathrm{mmHg})\end{array}$} & \multirow{2}{*}{$\begin{array}{c}\text { RER Peak } \\
\left(\mathrm{VCO}_{2} / \mathrm{VO}_{2}\right) \\
\end{array}$} & \multirow{2}{*}{$\begin{array}{c}\text { Workload } \\
\text { Peak (watts) }\end{array}$} & \multirow{2}{*}{$\begin{array}{l}\mathrm{VO}_{2} \text { at VAT } \\
(\mathrm{ml} / \mathrm{kg} / \mathrm{min})\end{array}$} & \multirow{2}{*}{$\begin{array}{l}\text { Workload at } \\
\text { VAT (watts) }\end{array}$} & \multirow{2}{*}{$\begin{array}{l}\mathrm{HR} \text { at VAT } \\
\text { (beats/min) }\end{array}$} \\
\hline & Measured & Percent Predicted & & Measured & Percent Predicted & & & & & & \\
\hline 0 & 23.7 & $72.0 \%$ & 49.3 & 158 & $86.8 \%$ & 115 & 1.26 & 87 & 12.6 & 40 & 127 \\
\hline 15 & 26.2 & $79.6 \%$ & 47.7 & 171 & $94.0 \%$ & 134 & 1.26 & 91 & 13.1 & 35 & 134 \\
\hline 55 & 24.8 & $75.4 \%$ & 73.4 & 175 & $96.2 \%$ & 142 & 1.58 & 110 & 14.6 & 50 & 129 \\
\hline 92 & 26.9 & $81.2 \%$ & 65.7 & 178 & $97.8 \%$ & 138 & 1.44 & 111 & 16.1 & 70 & 141 \\
\hline 125 & 29.7 & $90.3 \%$ & 77.8 & 180 & $98.9 \%$ & 138 & 1.38 & 116 & 18 & 61 & 138 \\
\hline 180 & 28.2 & $85.7 \%$ & 88.4 & 177 & $97.3 \%$ & 152 & 1.52 & 120 & 15.6 & 50 & 133 \\
\hline 248 & 30.1 & $91.4 \%$ & 88.5 & 173 & $95.1 \%$ & 160 & 1.56 & 120 & 17.4 & 60 & 133 \\
\hline 317 & 32.3 & $98.2 \%$ & 95.2 & 173 & $95.1 \%$ & 154 & 1.38 & 126 & 18.8 & 50 & 127 \\
\hline 420 & 30.6 & $93.0 \%$ & 89.6 & 186 & $102.2 \%$ & 174 & 1.47 & 120 & 16.8 & 50 & 139 \\
\hline 675 & & & & & & & & & & & \\
\hline (Last infusion) & 25.4 & $77.2 \%$ & 85.4 & 189 & $103.8 \%$ & 166 & 1.44 & 120 & 14.5 & 44 & 142 \\
\hline 708 & & & & & & & & & & & \\
\hline (30 days off saline) & 24.6 & $74.8 \%$ & 65.2 & 173 & $95.1 \%$ & 154 & 1.35 & 116 & 12.2 & 41 & 121 \\
\hline
\end{tabular}

Abbreviations: $\mathrm{VO}_{2}$ : volume of oxygen consumed; $\mathrm{VCO}_{2}$ : volume of carbon dioxide; $\mathrm{V}_{\mathrm{E}}$ : minute ventilation; HR: heart rate; SBP: systolic blood pressure; RER: respiratory exchange ratio; VAT: ventilatory anaerobic threshold. 
predicted) before the first infusion to a maximum of $32.3 \mathrm{ml} / \mathrm{kg} / \mathrm{min}$ (98\% of age-predicted). Similarly, peak HR showed a general linear improvement from 158 beats/min before the first infusion (87\% of agepredicted) to 189 beats/min (104\% of age-predicted). In addition, SBP at peak exercise also increased from $115 \mathrm{mmHg}$ before the first infusion to 174 $\mathrm{mmHg}$ after 420 days of infusion. The patient's chief symptom of brain fog had reduced steadily throughout treatment, and the patient's self-reported recovery time from CPET reduced from 5 days to 1-2 days by the end of treatment. The number of symptoms recorded after CPET also reduced. At Day 675 (conducted after the last infusion) and Day 708 (30 days after the last infusion), a decrease in $\mathrm{VO}_{2}$, was noted at peak exertion and ventilatory anaerobic threshold. The decrease began before cessation of IV saline treatments, and the magnitude of decrease approached pre-infusion levels for $\mathrm{VO}_{2}$. However, HR and SBP remained above pre-infusion levels, and the patient's symptoms remained improved overall. The patient was unable to resume her pre-illness level of occupational activity at the end of the intervention period, but she reported that her quality of life had improved due to resolution of her chief symptom of "brain fog."

\section{Discussion}

While many researchers have identified the autonomic nervous system as a potential cause of dysfunction in ME/CFS [19]. the mechanism of this abnormal sympathetic response is not well-described in the literature. Boneva et al. [20] revealed that individuals with ME/CFS exhibit significantly elevated resting HR and decreased heart rate variability (HRV) during sleep as compared to controls, and attributed both to a state of sympathetic hyperactivity characteristic of ME/CFS. This same pattern of HR and HRV was corroborated by Beaumont et al. [9] and was further linked to symptoms of cognitive impairment in people with ME/CFS. Additional research has implicated abnormal genetic expression in people with ME/CFS of cellular receptors involved in autonomic nervous system response, and have correlated this abnormality with reports of fatigue and myalgia [21]. Paradoxically, despite elevated heart rates at rest, patients with ME/CFS have decreased heart rates at peak and VAT compared to matched controls [11], which is further evidence of autonomic dysregulation in ME/CFS. This autonomic dysregulation is sufficiently prevalent in the ME/CFS population that the International Consensus Criteria acknowledges it as a contributor by way of symptoms of fatigue, pain, and abnormal cardiovascular performance [1].

These results indicate that regular isotonic saline administration may correct abnormal sympathetic activation at the onset of exercise. The patient's chronic low blood pressure may indicate hypovolemia, which would contribute negatively to ventricular filling both at rest and during exercise. Correcting volume status via IV saline administration may improve ventricular filling (directly with volume and indirectly via activation of the renin-angiotensin-aldosterone system), which could increase end diastolic volume. Frank-Starling's Law for cardiac function dictates that an increase in end diastolic volume will result in a more optimal lengthtension relationship of the cardiac myocytes, thus improving stroke volume by increasing cardiac contractility [22]. Such an improvement in contractile efficiency for the heart will improve systemic and central perfusion. Additionally, the sodium load may stimulate central nervous system sites associated with cardio-acceleration and hyperpnea that facilitate the exercise responses. This putative mechanism for improvement in exercise capacity appeared to benefit the patient in terms of sustained improvements in HR and SBP at peak and VAT.

Paradoxically, the patient demonstrated a reduction in $\mathrm{VO}_{2}$ before cessation of IV saline treatments. The mechanism for this $\mathrm{VO}_{2}$ reduction was unclear. It was notable that the improvements in $\mathrm{HR}_{\text {peak }}$, $\mathrm{WL}_{\text {peak }}, \mathrm{VO}_{2 \mathrm{VAT}}, \mathrm{HR}_{\mathrm{VAT}}$, and $\mathrm{WL}_{\mathrm{VAT}}$ were maintained throughout the intervention period. Yet, these values declined after the treatment stopped, suggesting the IV saline treatment may have been associated with those observed improvements. The main physiological effects of daily IV saline infusions may occur within the first year, because of the observed plateau between 317-420 days of treatment. In this case, symptomatic improvement was maintained despite the observed plateau in physiological improvement. The patient's chief symptom or "brain fog" remained improved, which increased the patient's self-perceived quality of life. Recovery time also was reduced to 2 days after each CPET from 5 days at baseline. Future controlled studies should evaluate the potential for similar improvements in other people with ME/CFS, including for people with ME/CFS who also have POTS. 
Casework is helpful to demonstrate the clinical application of concepts and to generate research hypotheses. However, there are several important limitations to this work. The potential safety, as well as duration and dosage of saline to promote beneficial clinical effects, remain unknown. There are risks associated with the treatment described in this case study. The patient in this case received infusions through a central venous line, which required a specialist for placement and frequent care. In addition, bacteremia is a known risk associated with IV treatment. These risks must be measured carefully in the context of well controlled studies. It is unknown whether the observed improvements in exercise capacity would generalize to other individuals with ME/CFS. Also, the potential mechanism of effects on exercise capacity of saline administration remain unknown, and specific data regarding patient functional, including occupational, responses to saline administration are still unavailable. Although the administration of IV saline to a patient with ME/CFS appeared promising to improve cardiometabolic functioning, symptoms, and activities of daily living in this case, these important remaining gaps in our understanding should be the subject of adequately designed controlled trials.

\section{Conclusion}

The functional burden of ME/CFS on patients is immense. Mechanistic research aimed at identifying effective evidence-based treatments is essential. This case study represents an introductory probe into both a potential explanation for the abnormal exercise response observed with ME/CFS, and a potential intervention to provide relief through amelioration of abnormal heart rate responses to exercise. While intravenous delivery may be invasive, saline administration offers the benefit of little to no side effects as compared to other pharmaceutical interventions. Additionally, it may improve physiological responses to exercise. Improving exercise response and maximizing the available workload for individuals with $\mathrm{ME/CFS}$, in turn, could disrupt the cycle of functional decline that is frequently associated with the condition. Observations from this case study may warrant deeper investigation in the context of larger, randomized studies that are designed to establish cause-effect relationships between IV saline administration, cardiac responses, and potential symptomatic benefit in people with ME/CFS.

\section{Acknowledgments}

At the time of writing, Mr. Ward was a student physical therapist in the Department of Physical Therapy at University of the Pacific.

\section{Conflict of interest}

The authors affirm they have no conflicts of interest related to the content presented in this manuscript.

\section{References}

[1] Carruthers BM, van de Sande MI, De Meirleir KL, Klimas NG, Broderick G, Mitchell T, et al. Myalgic encephalomyelitis: International Consensus Criteria. J Intern Med. 2011;270(4):327-38.

[2] Fukuda K, Straus SE, Hickie I, Sharpe MC, Dobbins JG, Komaroff A. The chronic fatigue syndrome: a comprehensive approach to its definition and study. International Chronic Fatigue Syndrome Study Group. Ann Intern Med. 1994;121(12):953-9.

[3] Kingdon CC, Bowman EW, Curran H, Nacul L, Lacerda EM. Functional Status and Well-Being in People with Myalgic Encephalomyelitis/Chronic Fatigue Syndrome Compared with People with Multiple Sclerosis and Healthy Controls. Pharmacoecon Open. 2018;2(4): 381-92.

[4] Nacul LC, Lacerda EM, Pheby D, Campion P, Molokhia M, Fayyaz S, et al. Prevalence of myalgic encephalomyelitis/chronic fatigue syndrome (ME/CFS) in three regions of England: a repeated cross-sectional study in primary care. BMC Med. 2011;9:91.

[5] Collin SM, Crawley E, May MT, Sterne JA, Hollingworth W, Database UCMNO. The impact of CFS/ME on employment and productivity in the UK: a cross-sectional study based on the CFS/ME national outcomes database. BMC Health Serv Res. 2011;11:217.

[6] Ross SD, Estok RP, Frame D, Stone LR, Ludensky V, Levine CB. Disability and chronic fatigue syndrome: a focus on function. Arch Intern Med. 2004;164(10):1098-107.

[7] Shlaes JL, Jason LA, Ferrari JR. The development of the Chronic Fatigue Syndrome Attitudes Test. A psychometric analysis. Eval Health Prof. 1999;22(4):442-65.

[8] Hulens M, Rasschaert R, Vansant G, Stalmans I, Bruyninckx F, Dankaerts W. The link between idiopathic intracranial hypertension, fibromyalgia, and chronic fatigue syndrome: exploration of a shared pathophysiology. J Pain Res. 2018;11:3129-40.

[9] Beaumont A, Burton AR, Lemon J, Bennett BK, Lloyd A, Vollmer-Conna U. Reduced cardiac vagal modulation impacts on cognitive performance in chronic fatigue syndrome. PLoS One. 2012;7(11):e49518.

[10] VanElzakker MB. Chronic fatigue syndrome from vagus nerve infection: a psychoneuroimmunological hypothesis. Med Hypotheses. 2013;81(3):414-23.

[11] Davenport TE, Lehnen M, Stevens SR, VanNess JM, Stevens J, Snell CR. Chronotropic Intolerance: An Overlooked Determinant of Symptoms and Activity Limitation 
in Myalgic Encephalomyelitis/Chronic Fatigue Syndrome? Front Pediatr. 2019;7:82.

[12] Ruzieh M, Baugh A, Dasa O, Parker RL, Perrault JT, Renno A, et al. Effects of intermittent intravenous saline infusions in patients with medication-refractory postural tachycardia syndrome. J Interv Card Electrophysiol. 2017;48(3):255-60.

[13] Stevens S, Snell C, Stevens J, Keller B, VanNess JM. Cardiopulmonary Exercise Test Methodology for Assessing Exertion Intolerance in Myalgic Encephalomyelitis/Chronic Fatigue Syndrome. Front Pediatr. 2018;6:242.

[14] Vanness JM, Stevens SR, Bateman L, Stiles TL, Snell CR. Postexertional Malaise in Women with Chronic Fatigue Syndrome. J Womens Health (Larchmt).

[15] Sorensen B, Streib JE, Strand M, Make B, Giclas PC, Fleshner $\mathrm{M}$, et al. Complement activation in a model of chronic fatigue syndrome. J Allergy Clin Immunol. 2003;112(2):397-403.

[16] Whistler T, Jones JF, Unger ER, Vernon SD. Exercise responsive genes measured in peripheral blood of women with chronic fatigue syndrome and matched control subjects. BMC Physiol. 2005;5(1):5.

[17] LaManca JJ, Sisto SA, DeLuca J, Johnson SK, Lange G, Pareja J, et al. Influence of exhaustive treadmill exercise on cognitive functioning in chronic fatigue syndrome. Am J Med. 1998;105(3A):59S-65S

[18] American College of Sports Medicine. ACSM's Guidelines for Exercise Testing and Prescription. Baltimore, MD, USA: Lippincott Williams Wilkins; 2006.

[19] Newton JL, Okonkwo O, Sutcliffe K, Seth A, Shin J, Jones DE. Symptoms of autonomic dysfunction in chronic fatigue syndrome. QJM. 2007;100(8):519-26.

[20] Boneva RS, Decker MJ, Maloney EM, Lin JM, Jones JF, Helgason HG, et al. Higher heart rate and reduced heart rate variability persist during sleep in chronic fatigue syndrome: a population-based study. Auton Neurosci. 2007;137(12):94-101.

[21] Light AR, White AT, Hughen RW, Light KC. Moderate exercise increases expression for sensory, adrenergic, and immune genes in chronic fatigue syndrome patients but not in normal subjects. J Pain. 2009;10(10):1099-112.

[22] Delicce AV, Basit H, Makaryus AN. Physiology, Frank Starling Law Treasure Island (FL): StatPearls Publishing; 2019. Available from: https://www.ncbi.nlm. nih.gov/books/NBK470295/ 